\title{
CU-Boulder Center for Cryo-Electron Microscopy (CCET)
}

\author{
Andreas Hoenger
}

University of Colorado, Boulder CO, Boulder, Colorado, United States

The CU-Boulder Center for Cryo-Electron Tomography (CCET) one of four partners in the newly formed NIH consortium called "The National Network for Cryo-ET", composed of a central Hub (The Midwest Center for Cryo-ET, Madison WI, The National Center for in-situ Tomographic Ultramicroscopy, New York NY, and the Stanford-SLAC Cryo-ET Specimen Preparation Center, Stanford CA). Our lab is located in Boulder, Colorado. We are building on the experience of a former P41 facility for cellular electron tomography. The specialties of our center is a long-standing tradition in cellular 3-D electron microscopy (plastic-embedding, and more recently cryo-ET). We will take advantage of two already existing EM facilities, our MCDB departmental EM service unit, and the HHMI funded Titan-Krios facility in our Biochemistry Department (BioKEM). Hence, we have three interacting facilities optimizing specimen preparation processes, technology developments, and data collection.

The Boulder CCET will place a particular effort towards solving the challenges that emerge with navigating a complex cytosol. While large structures usually are quite easy to identify, even in the crowded environment of a frozen cell or organelle, smaller complexes or thin filaments are usually much harder to find, but that is where the details are. To this end we will focus on correlative light and electron microscopy methods, the implementation of electron-dense labels with high spatial resolution, as well as light-activation of processes right before the vitrification of specimens. At Boulder, we offer a wide range of EM-related methods for 3-D visualization of cells and large organelles, including FIB-SEM lamella production, vitrified sectioning, and also plastic-embedding for preliminary studies.

Both departments that are involved in this center have ample experience with many cellular and biochemical issues. This offers access to quick help and advice with incoming projects. The Center will also offer training in specimen preparation and/or data acquisition, and, if desired, will help outside groups to implement these technologies in their own labs. 